\title{
Emerging Alternatives to Conventional Clinic Visits in the Era of COVID-19: Adoption of Telehealth at VCU Adult Cystic Fibrosis Center
}

This article was published in the following Dove Press journal: International Journal of General Medicine

\section{Caitlin Womack (D) \\ Ruhan Farsin (D) \\ Mahsa Farsad \\ Nauman Chaudary (D) \\ Division of Pulmonary Disease and Critical Care Medicine, Department of Medicine, Virginia Commonwealth University, Richmond, VA, USA}

Correspondence: Caitlin Womack; Nauman Chaudary

Email womackce@mymail.vcu.edu; nauman.chaudary@vcuhealth.org

\begin{abstract}
Cystic fibrosis (CF) is a genetic disease in which consistent follow-up care is required to avoid a decline in pulmonary and nutritional health. It is believed that if a CF patient ceases treatment for 2 days, this can result in an exacerbation. One week of missed treatments can result in a hospitalization and 1 month of missed treatments can result in an earlier demise. With a global pandemic that has affected more than 9 million people, many $\mathrm{CF}$ clinics were required to take steps to avoid transmission of this dangerous virus. This may result in delays in delivery of timely CF care due to closure of clinics and pulmonary function testing (PFT) laboratories and limited staff allowed on site for conducting in-person visits. These measures, along with suggestions from the Cystic Fibrosis Foundation (CFF) to extend the social distancing longer than traditional CDC recommendations for the $\mathrm{CF}$ community, create an urgent need to explore novel ways to deliver safer care via new standards in chronic health conditions like CF. Especially, as these preventive strategies may be necessary for long-term maintenance, few objective alternatives exist to guide clinicians and allied health professionals in CF centers how to proceed in this new era. This also presents an opportunity for novel approaches that could improve delivery of $\mathrm{CF}$ care with remote monitoring and real-time delivery of care in patients' home environments. Such emerging approaches could benefit patient care, leading to reduced costs and readmissions and improved access to care, medication adherence, and patient communication. We summarize our own experience and discuss the emerging delivery of CF care which can be generalizable to other pulmonary illnesses.
\end{abstract}

Keywords: coronavirus, COVID-19, telehealth, cystic fibrosis

\section{Introduction}

Cystic fibrosis (CF) is a complex, multi-system disorder characterized by an autosomal recessive mutation in the cystic fibrosis transmembrane regulator (CFTR) gene. Patients with CF experience significant morbidity and mortality with a myriad of multi-organ manifestations which may include the lungs, pancreas, liver, gastrointestinal and reproductive tracts, and bones. ${ }^{1}$ Due to this complexity, the Cystic Fibrosis Foundation (CFF) recommends that patients are seen at least quarterly for screening and management, including at least two respiratory cultures and pulmonary function tests (PFTs) per year. ${ }^{2}$ Studies have likewise suggested that more frequent monitoring of $\mathrm{CF}$ patients is associated with improved outcomes. Johnson et al demonstrated that more frequent visits, spirometry measurements of lung function, and cultures for respiratory pathogens were associated with improved 
lung health. ${ }^{3}$ Likewise, nutritional status should be routinely assessed every 3 months to allow for swift intervention when necessary. ${ }^{4}$ The literature additionally supports adoption of a multidisciplinary care model for patients with CF in order to best manage the multi-system manifestations of the disorder. ${ }^{1,4,5}$ There are more than 30,000 individuals living with $\mathrm{CF}$ in the United States but only 130 CFF-accredited centers specialized in delivering multidisciplinary care for $\mathrm{CF}^{6}{ }^{6}$ Therefore, many patients must travel to reach their closest center, in the process incurring expenses associated with transportation, lodging, childcare, and lost income.

\section{Impact of COVID-19 on CF Center Care}

The coronavirus 2019 (COVID-19) pandemic necessitated a brisk transition to telehealth worldwide in a manner unprecedented in its immediacy and extent. Although telehealth has been deployed in previous crises, including during the 2003 severe acute respiratory syndrome (SARS) pandemic in China, severe droughts in Australia, and hurricanes Irma and Harvey in the United States, previous uptake of telehealth in emergency situations has been comparatively limited. ${ }^{7}$ In March 2020, previously imposing restrictions on telehealth coverage and reimbursement were promptly relaxed as hospitals across the United States began to restrict in-person clinical encounters, and within days the utilization of telehealth increased precipitously. ${ }^{8}$ The number of telehealth visits at a single academic institution in Ohio increased from less than 100 visits per day to over 2200 daily visits over 24 days following the start of the pandemic. ${ }^{9}$ Physical distancing is particularly important for patients with CF who may be predisposed to severe manifestations of COVID-19 due to their comorbidities ${ }^{10}$ and for whom recommendations for strict infection control have long been in place even prior to COVID-19. ${ }^{11}$

\section{Potential Use of Telehealth in CF Centers During COVID-19}

Telehealth has been explored as a way to circumvent these barriers to care while still ensuring that patients receive the good quality, multidisciplinary care and frequent monitoring that has been associated with good outcomes. Telehealth refers to the delivery of healthcare remotely through phone call or video conference that may include utilization of telemonitoring devices and protocols. As technology has advanced, telehealth has gained recognition; nevertheless, barriers such as provider disinterest in telehealth adoption, ${ }^{7}$ diminished reimbursement, ${ }^{12}$ state licensure requirements, ${ }^{13}$ and limited at-home patient access to appropriate technology (ie internet-enabled devices, high-speed internet) ${ }^{14}$ have slowed the rate of uptake. While telehealth utilization did significantly increase from 2005 to 2017, use remained infrequent by $2017 .^{15}$ Still, telehealth has been investigated in the context of CF care as a way to enhance convenience, decrease cost, fill gaps in access due to geography, and increase the frequency of monitoring to improve patient satisfaction and outcomes. ${ }^{16,17}$ The few studies investigating patient experience with telehealth have demonstrated high satisfaction and good uptake, indicating this to be a feasible option for patients. ${ }^{18,19}$ A recent review determined telehealth services generally to be equivalent to in-person care in most disciplines, ${ }^{20}$ and although there is a need for more longitudinal comparative studies, specialty teleconsultation services have demonstrated equivalence to face-toface patient care during the COVID-19 pandemic. $^{21}$ However, there is little research into long-term outcomes associated with telehealth care. ${ }^{22}$

\section{The Role of Telehealth at VCU CF Center in CF Care to Reduce the Spread of COVID-19}

Virginia Commonwealth University (VCU) Adult Cystic Fibrosis Center followed nationwide trends, following institutional guidelines in transitioning to telehealth visits starting in early March 2020. The VCU CF center serves 104 adult patients in central Virginia. The center is accredited by the CFF and adopts an interdisciplinary approach to patient care involving a physician, nurse, dietitian, respiratory therapist, social worker, and pharmacist. Medical collaboration involves pulmonology, endocrinology, gastroenterology, and psychiatry, among other specialties as indicated. Patients are followed to ensure they receive annually a minimum of 4 visits, 2 PFTs, 2 cultures, a lab draw and chest X-ray, colonoscopy, nutrition assessment, and mental health screening per year, along with routine colon and bone density screenings, as per CFF recommendations.

The protocol in transitioning the CF center to telehealth was carefully designed to ensure uninterrupted access to patient-centered multidisciplinary care. Our center participated in the Cystic Fibrosis Learning Center (CFLN) COVID-19 Telehealth Innovation Lab (iLab), a collaborative initiative focused on utilizing established 
quality improvement tools, including Plan Do Study Act (PDSA) cycles and Failure Modes and Effects Analysis (FMEA), to develop and refine a telehealth protocol for $\mathrm{CF}$ care during the pandemic. Through weekly meetings with participating CF centers and monthly data collection of outcome measures starting in May 2020, our center refined the telehealth protocol with the goal of implementing telehealth visits that are as close as possible to face-to-face visits. PDSA and FMEAs helped us to identify the differences between the two models to close the gap as much as possible. In an effort to create this model we included co-production and agenda setting, which involve eliciting patient and family expectations for the visit during the pre-visit planning phase. As the center implemented telehealth, patients were also provided an 11-question survey in an effort to understand patient experience and drive quality improvement with this novel care model.

There is very limited guidance available to the clinical team to adapt to these emerging trends in the delivery of health care with these new platforms. As health systems across the United States embrace emerging trends in telehealth, we present our single-center experience of VCU Adult CF Center in transitioning from in-person clinic visits to telehealth for routine $\mathrm{CF}$ care in response to the
COVID-19 pandemic. We will discuss the protocol developed and followed by the center and the patient experience with telehealth in this setting. These methods may be compared to protocols developed at other CF clinics amidst this public health crisis ${ }^{20}$ and may be of use to other CF centers or to any other multidisciplinary clinics needing to quickly implement telehealth services. It is our intent that lessons from our institution's experience and patient views may serve as a powerful tool to inform best practices for telehealth provision that extend beyond the global pandemic.

\section{Methods}

\section{Pre-Clinic (Figure I)}

All clinic visits scheduled prior to the pandemic were converted to virtual visits. In addition, a triage system was implemented for new appointments (Table 1). Four weeks prior to the clinic day, a team member calls patients who are scheduled to be seen at the clinic to confirm their appointment, verify their access to the portal and Zoom, and troubleshoot technology if necessary. If patients need to see other providers, appointments will be coordinated to occur on the same day. We currently have two providers, an endocrinologist and a psychiatrist, who coordinate their clinics with the CF clinic.

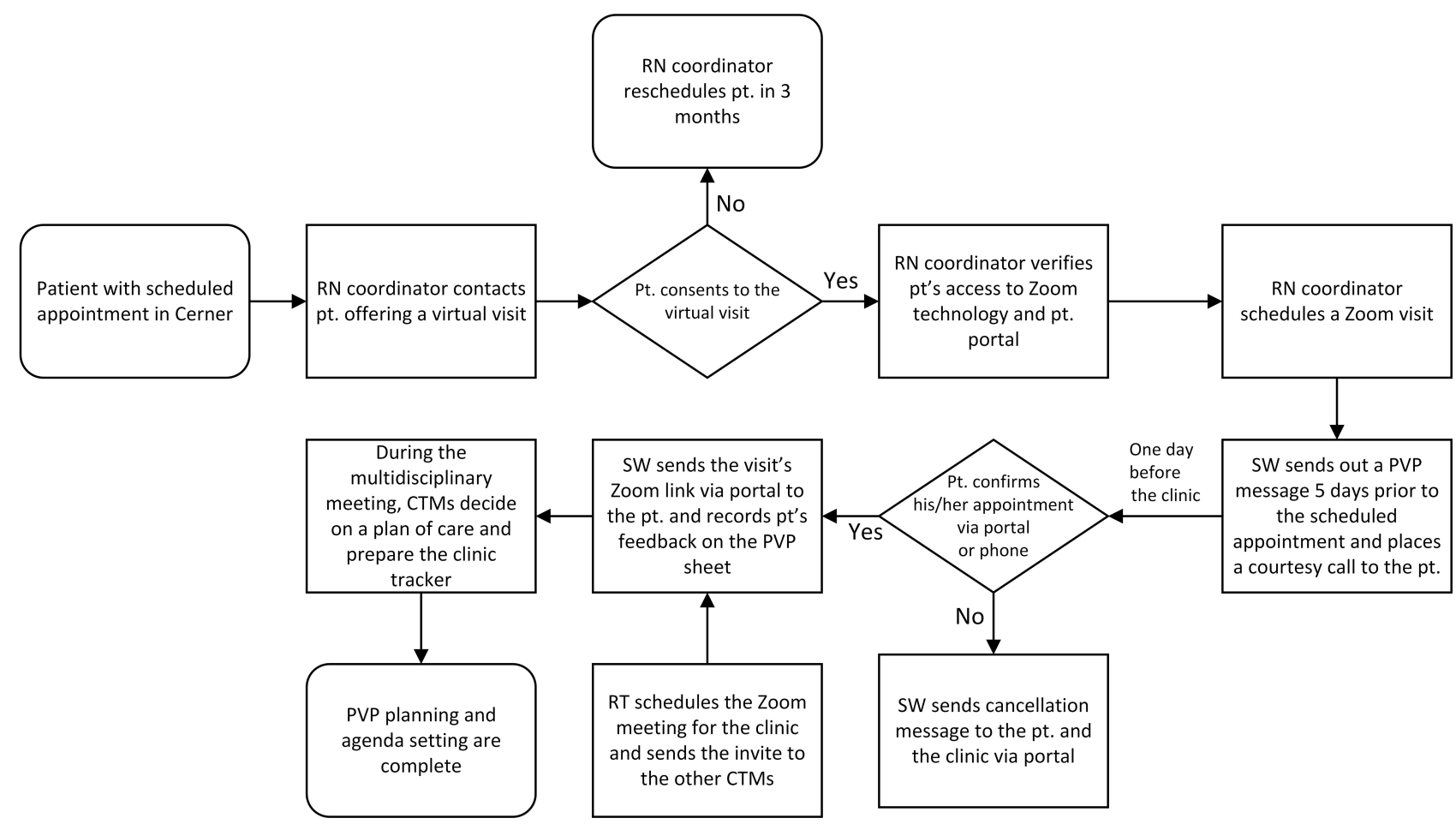

Figure I Pre-visit planning process. The process followed by cystic fibrosis care team members to schedule patients for virtual visits, set agenda in collaboration with the patients, and collaboratively prepare a plan of care prior to the clinic visit.

Abbreviations: RN, registered nurse; pt., patient; SW, social worker; RT, respiratory therapist; CTM, care team member; PVP, pre-visit planning. 
Table I VCU Adult CF Center Virtual Visit Appointment Classification During COVID-19. New or Follow-Up Appointments for Virtual Visits are Scheduled Based on the Criteria in This Table

\begin{tabular}{|c|c|}
\hline $\begin{array}{l}\text { Virtual Visit } \\
\text { Classification }\end{array}$ & Details \\
\hline Needs to be seen soon & $\begin{array}{l}\text { Patients with active issues } \\
\text { - Sick visit } \\
\text { - On IV antibiotics at home } \\
\text { - Recently d/c from the hospital } \\
\text { - Active treatment for NTM, ABPA } \\
\text { - Decline in lung function, recent oral } \\
\text { antibiotics, and who are having close } \\
\text { follow-ups } \\
\text { - Referral to transplant center in } \\
\text { process } \\
\text { - Lung transplant follow-up } \\
\text { - Poor nutritional status }\end{array}$ \\
\hline $\begin{array}{l}\text { Needs to be seen within } \\
4-6 \text { weeks }\end{array}$ & $\begin{array}{l}\text { Patient with advanced lung disease } \\
\text { who have been clinically stable in the } \\
\text { last } 3 \text { months }\end{array}$ \\
\hline $\begin{array}{l}\text { Appointment could be } \\
\text { rescheduled in } 2 \text { months }\end{array}$ & $\begin{array}{l}\text { Patients who do not meet the above } \\
\text { or below criteria }\end{array}$ \\
\hline $\begin{array}{l}\text { Appointment could be } \\
\text { rescheduled in } 3-4 \text { months }\end{array}$ & $\begin{array}{l}\text { Patients with no active issues: } \\
\text { - Stable lung function and infrequent } \\
\text { exacerbations (<1/year) and good } \\
\text { nutritional status } \\
\text { - Patients scheduled for quarterly fol- } \\
\text { low-up } \\
\text { - CFRD clinic } \\
\text { - CF Mental Health Clinic }\end{array}$ \\
\hline
\end{tabular}

Abbreviations: IV, intravenous; d/c, discharged; NTM, nontuberculous mycobacterial; ABPA, allergic bronchopulmonary aspergillosis; CFRD, cystic fibrosis-related diabetes.

Five days prior to the clinic visit, a pre-visit planning (PVP) message is sent to patients who are scheduled to be seen at the clinic. This message includes the date and time of the visit, team members who wish to see the patient, and inquiries regarding patient priorities and requests for the upcoming visit. In addition, a courtesy call is made to patients to inform them of this important message in the patient portal. Patients have 4 days to respond to the portal message and confirm their appointment.

To reduce clinic "no show" rates, appointments of patients who fail to confirm their appointment by the deadline are canceled. A portal message is sent to the patient and the clinic to inform them of the appointment cancellation.

One day prior to the clinic visit, the respiratory therapist (RT) serves as the Zoom host to schedule the Zoom meeting for the clinic and shares the Zoom link with the other team members. Patients who confirmed their appointment receive a portal message containing their appointment time, the Zoom link for their appointment, and instructions for a successful meeting via Zoom.

Also, 1 day prior to the clinic day, team members discuss each scheduled patient in a multidisciplinary meeting. During this meeting, a pre-visit plan is created based on specific data provided by each team member and feedback received from patients in response to the PVP message. All these data are recorded on a pre-visit planning sheet shared in Microsoft One-Drive between team members. In addition, a clinic tracker (Table 2) is filled with this necessary information for management of clinic flow.

\section{Clinic Day}

Prior to each encounter, the host on Zoom creates breakout rooms for each patient (to ensure HIPAA compliance) and a huddle room for the $\mathrm{CF}$ team. A nurse from the clinic calls scheduled patients to complete the check-in process. This includes completion of a questionnaire in the electronic medical record. This questionnaire gathers data regarding travel history, fall risk, cognition, abuse and neglect, flu and pneumococcal screening, vital signs (temperature, respiratory rate, oxygen saturation, blood pressure), anthropometric data (weight, height), pain, peripheral IV, and respiratory assessment. All these data are self-reported by the patients.

The Zoom host admits patients to the virtual clinic (Zoom meeting room), greets patients, and presents patients with the itinerary for their upcoming visit. The patients are then transferred to their specific breakout rooms. The host, via a Microsoft Teams message, informs the team that the patient is ready for his/her visit. Flow is regulated via a clinic tracker (Table 3 ) and communications on Microsoft Teams. This virtual clinic flow is represented in Figure 2.

Zoom breakout rooms therefore provide the opportunity for multidisciplinary visits, while Microsoft Teams facilitates communication between team members and the clinic tracker regulates clinic flow.

\section{Post-Visit}

A summary of the finalized care plan, which is the result of patient and team collaboration, is posted on the clinic tracker and pre-visit planning sheet to coordinate care and follow-up. The team discusses the process, implementation, and opportunities for improvement during the weekly quality improvement meeting. 


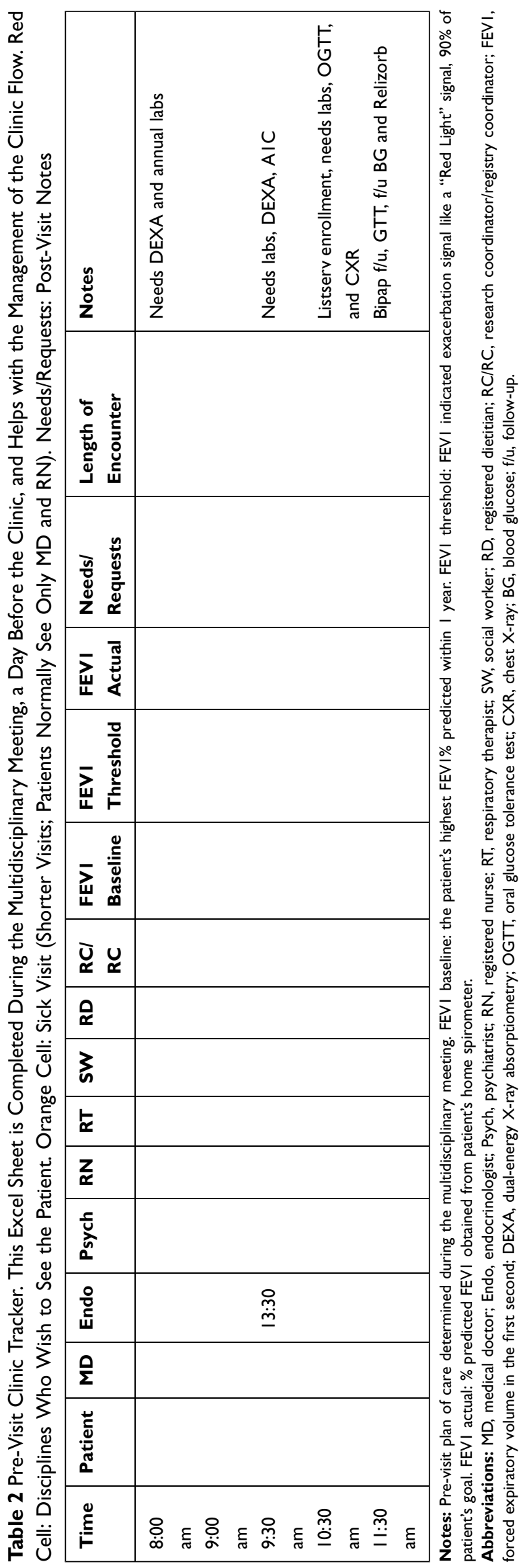

\section{Labs, Imaging, PFTs, and Cultures}

In addition to the virtual visit as a substitute for in-person encounters, alternatives to traditional labs, imaging, PFTs, and culture collection have also been implemented to further reduce the risk of exposure to COVID-19.

More rigorous safety precautions have been put in place for CF patients who must go in-person to the $\mathrm{CF}$ clinic for labs, imaging, cultures, and PFTs.

All patients who are scheduled for labs, chest X-ray, or PFTs call the clinic on arrival and check in on the phone. Once check-in is complete, a clinic RN greets them at the entrance, checks their temperature, screens them for signs and symptoms of COVID-19, makes sure they wear a mask, and escorts them to the assigned room depending on their reason for the visit: either the lab and culture room, imaging room, or visit room for PFTs. One door is dedicated to entrance and another door to exit. There are no patients in the clinic's waiting area; all patients are escorted in and out of the clinic.

All surfaces and rooms are disinfected hourly.

All clinic employees are placed 6 feet apart and wear masks.

All healthcare members who encounter CF patients wear a gown and gloves.

Labs: bloodwork could be collected at home by a home health agency or at the clinic. A nurse coordinator arranges the blood collection at home.

Sputum and throat cultures: specimens could be collected at home and mailed to the lab or may be collected at the clinic (Figure 3).

PFTs: PFTs could be measured at home by patients using home spirometry or at the clinic. Since the start of the pandemic, VCU Adult CF Center purchased and mailed home spirometers to patients who confirmed the need for one. The adult CF center selected ZephyRx "MIR Spirobank Smart" as the portable (home) spirometer for patient use, which is approved by the CFF. Below is the process which was put in place by the CF clinic administrative assistant:

1. An initial email was sent through the Listserv to confirm patient addresses.

2. A spreadsheet was created including all CF patients in VCU Adult Center, their phone number, and physical and email addresses.

3. Patients were color coded based on their responses: 1. Yellow: confirmed address and awaiting spirometer. 


\begin{tabular}{|c|c|c|c|c|c|c|}
\hline 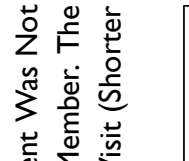 & $\begin{array}{l}\stackrel{y}{u} \\
\stackrel{0}{z}\end{array}$ & 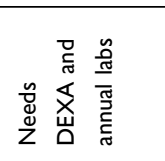 & & 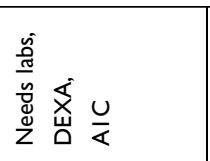 & 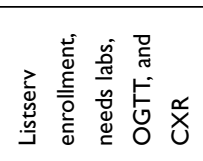 & 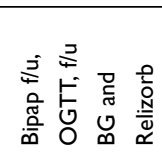 \\
\hline 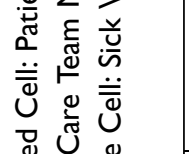 & 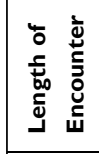 & 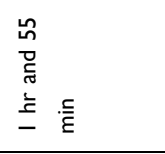 & & 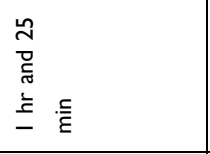 & $\begin{array}{l}\stackrel{\frac{c}{E}}{E} \\
\stackrel{m}{m}\end{array}$ & 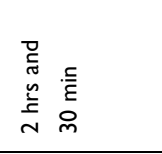 \\
\hline 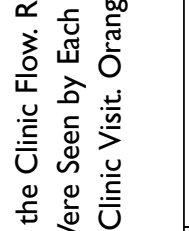 & 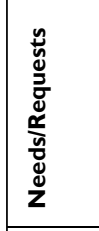 & 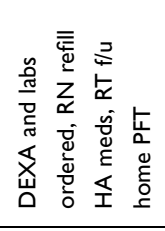 & & 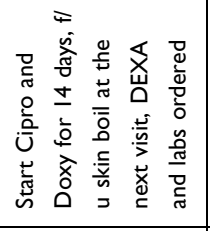 & 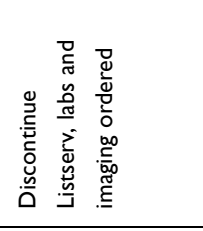 & 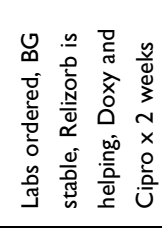 \\
\hline 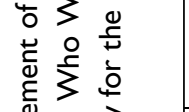 & 昰 & ○̊ & & $\begin{array}{l}\stackrel{0}{\circ} \\
\text { ¿े }\end{array}$ & ஓें & $\frac{\circ}{\sigma}$ \\
\hline 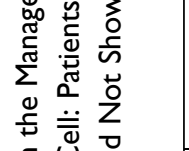 & 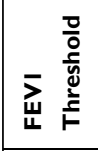 & $\stackrel{\circ}{\circ}$ & & ஓ్లి & ஓे & $\stackrel{\circ}{\stackrel{\circ}{\sigma}}$ \\
\hline 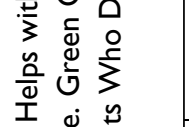 & 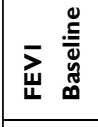 & ஓ̊ & & 总 & ڤે & ๖ेㅇํ \\
\hline & 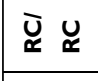 & & & & & \\
\hline 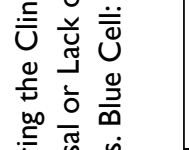 & จे & $\begin{array}{l}8 \\
\dot{0} \\
\stackrel{1}{\alpha} \\
2\end{array}$ & & 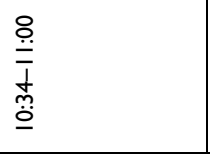 & & $\begin{array}{l}\stackrel{8}{+} \\
\stackrel{+}{i} \\
\stackrel{i}{\underline{m}}\end{array}$ \\
\hline 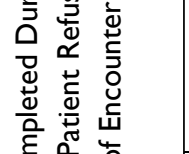 & 3 & $\begin{array}{l}\frac{0}{\dddot{q}} \\
\frac{1}{2} \\
\frac{\alpha}{\alpha}\end{array}$ & & & & 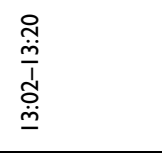 \\
\hline 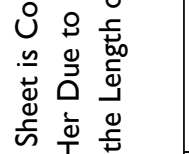 & $\underline{x}$ & & & & 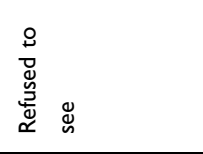 & 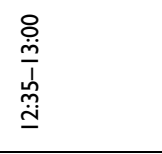 \\
\hline 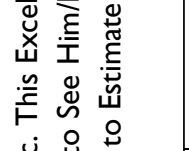 & $z_{x}$ & $\begin{array}{l}\frac{n}{\not i} \\
\dot{0} \\
\dot{\alpha}\end{array}$ & & 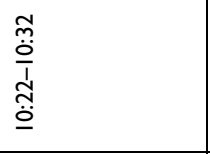 & 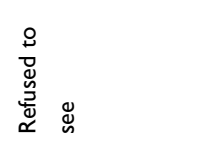 & 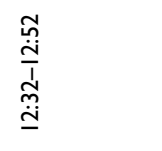 \\
\hline 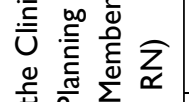 & $\begin{array}{l}\frac{5}{\bar{n}} \\
0 \\
0\end{array}$ & & & & & \\
\hline 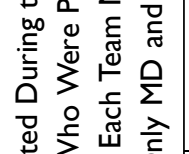 & 远 & & & $\begin{array}{l}\stackrel{o}{\dot{+}} \\
\frac{1}{1} \\
\stackrel{m}{\dot{m}}\end{array}$ & & \\
\hline 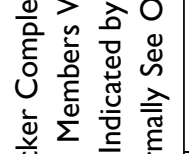 & $\frac{0}{\Sigma}$ & $\begin{array}{l}\text { 岁 } \\
\text { ơ }\end{array}$ & & 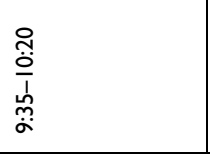 & 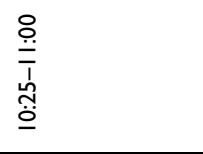 & $\begin{array}{l}\stackrel{O}{\vec{j}} \\
\bar{d} \\
\stackrel{m}{=}\end{array}$ \\
\hline 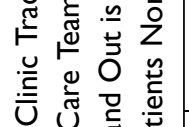 & 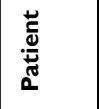 & & & & & \\
\hline 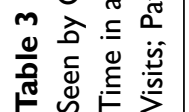 & $\stackrel{\mathscr{E}}{\xi}$ & 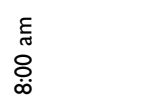 & 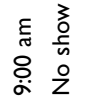 & $\begin{array}{l}\xi \\
\stackrel{్}{\sigma} \\
\stackrel{0}{\alpha}\end{array}$ & 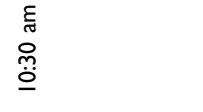 & 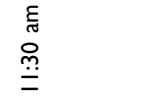 \\
\hline
\end{tabular}




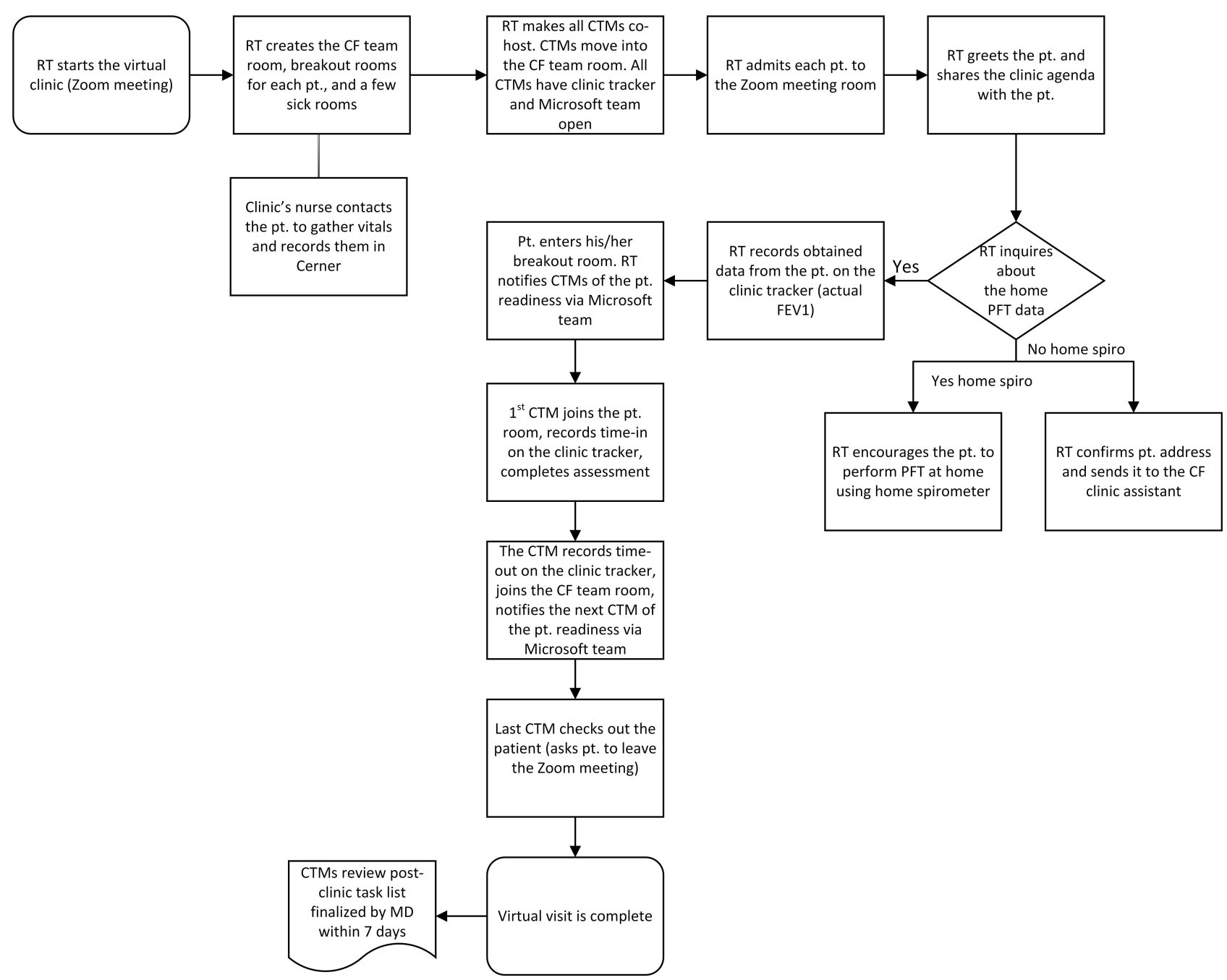

Figure 2 Virtual clinic flow chart. RT acts as a host for the Zoom visits, and creates breakout rooms for patients and a CF team room for CTMs. Each CTM meets with the patient in his/her breakout room for assessment and the last CTM checks out the patient from the clinic. Clinic flow is managed via clinic tracker and Microsoft Teams. Abbreviations: RT, respiratory therapist; CTM, care team member; pt., patient; PFT, pulmonary function test; CF, cystic fibrosis; MD, medical doctor.

2. Green: confirmed address and received spirometer.

3. Red: could not confirm address (no patient response).

4. In addition, the RT, when greeting patients on the Zoom meeting, confirms patients' addresses and transmits those to the administrative assistant.

5. In collaboration with the pulmonology division's fiscal tech, a FedEx account was created. This account is tied to VCU Adult CF Center funds and is used to create shipments and schedule a pickup.

6. The portable spirometer is shipped with two nose clips and an instruction letter via FedEx.

After a patient receives the portable spirometer, he or she is instructed to read the portable spirometer instruction letter to become familiar with the set-up, use, and maintenance (such as daily cleaning instructions) of the machine. In addition, the patient is instructed to practice using the portable spirometer for at least 5 days, once per day at the same time; for example, the patient may use the spirometer after the morning breathing treatments and airway clearance techniques (ACTs). Thereafter, the patient should have a good baseline of what his/her goal forced expiratory volume in 1 second relative to forced vital capacity (FEV1\%) will be with the portable spirometer. This value may be slightly lower than what the patient has established in previous clinic visits on the hospital-approved spirometer (pneumotachometer). This is based on the knowledge that the lung function tests are highly effort-dependent and there are no trained medical personnel in the home setting who can encourage the patient to perform the test with maximum effort. The patient's portable spirometer values are used as trending values (the highest value on the patient's portable 


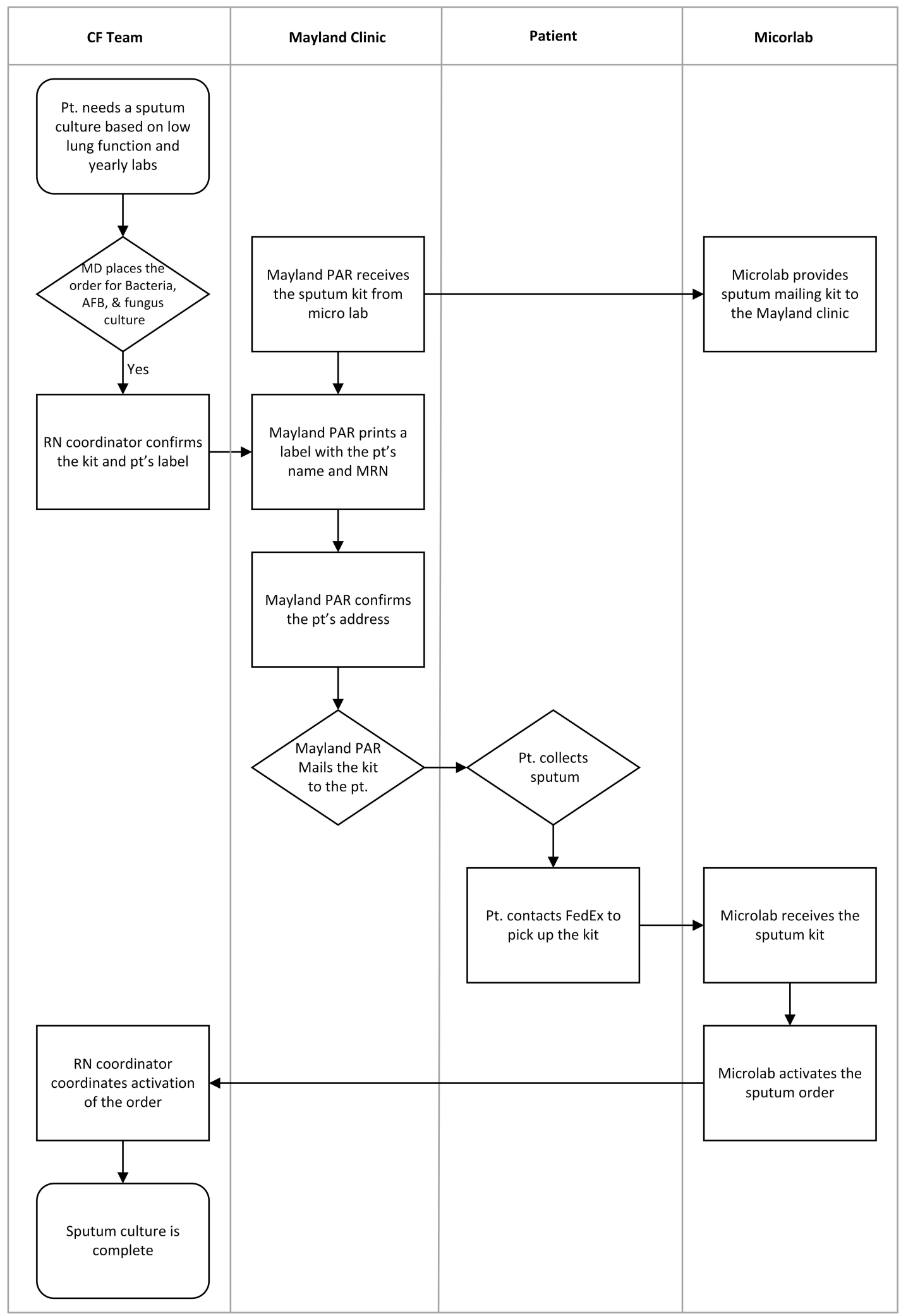

Figure 3 Home sputum and throat culture collection flow chart. Sputum culture is ordered by the MD. Nurse coordinator coordinates communication between the lab, the $\mathrm{MD}$, and the Mayland clinic. Patient collects the sputum following instructions in the kit and sends it to the lab via FedEx.

Abbreviations: MD, medical doctor; AFB, acid-fast bacillus; RN, registered nurse; PAR, patient access representative; MRN, medical record number. 
spirometer is the patient's portable goal FEV1\%). Thereafter, the patient is instructed to do the portable spirometer at least once per week, at the same time of the day (such as after the morning breathing treatments and ACTs). If the patient's FEV1 is declining and exacerbation symptoms are trending up, the patient is instructed to be vigilant by increasing breathing treatments and/or ACTs. In addition, the patient is instructed to notify the CF care team with concerns.

During virtual clinic visits, the RT records patients' FEV1 values on the clinic tracker; values for patients who share their portable spirometer values with the VCU Adult CF Center are available on the ZephyRx Provider Dashboard. Patients who do not share their FEV1 values provide the respiratory therapist (RT) with their most recent results including the date and time. In addition, they are instructed by the RT on how to share the portable spirometer results with the VCU Adult CF Center. The physician compares patients' recent results with their established goal FEV1\%. If the current results are less than $90 \%$ of the patients' goal FEV1\%, the physician would make clinical decisions accordingly.

\section{Patient Survey}

Sixty adult patients with CF seen in telehealth visits from April 1 to June 3, 2020 were provided with an 111question survey adapted from Cottage Health in California to assess patient views on telehealth care for $\mathrm{CF}$, including the potential impact on their rapport with providers and on their overall health. Patients were also asked about their interest in telehealth patient, family, and group education programs.

The surveys were sent immediately following telehealth visits over the patient portal in the electronic medical record. Surveys were sent to all patients seen through telehealth visits, and each patient was invited to fill out the survey once, even if that patient had multiple virtual visits over the given period of survey collection. Reminder messages and subsequent phone calls were sent 1 week later to patients who did not respond to the initial survey invitation. No identifying information was collected. Responses were recorded as either yes, no, or unsure in order to facilitate scoring for data analysis.

\section{Results}

Between April 1 and June 30, 2020, the VCU Adult CF Center conducted a total of 153 virtual visits: 125 via Zoom and 20 via phone. Out of 145 visits, 83 of those visits were synchronous meaning the visit included
Table 4 Number of Virtual Visits Conducted at the VCU Adult CF Center Between the Dates of April I and June 30, 2020 and Number of Clinic Visits Completed During the Same Period in 2019

\begin{tabular}{|l|l|l|}
\hline & $\begin{array}{l}\text { Virtual Visits } \\
\mathbf{2 0 2 0}\end{array}$ & Clinic Visits 2019 \\
\hline \# of clinic days & 12 & 9 \\
Total \# of pts & $106(104$ CF+2 CF & 104 (CF) \\
& eval) & 75 \\
Total \# of clinic visits & 83 & 40 \\
Total \# of pts seen & 53 & 15 \\
Pts with 2 visits & 13 & 2 \\
Pts with 3 visits & 4 & 2 \\
Pts with >3 visits & 2 & 64 (6 no show +58 \\
Pts with no clinic visits & $53(3$ no show +50 \\
& no appointments) & no appointments) \\
No show & 6 & 6 \\
\hline
\end{tabular}

Abbreviation: pt, patient.

a pulmonologist, other CF team members, and subspecialty providers when applicable (Table 4). Figure 4 shows the percentage of virtual visits conducted by each care team member at VCU Adult CF Center.

Overall survey responses indicated positive reception toward telehealth; $100 \%(\mathrm{n}=20)$ of surveyed patients felt there would be improved access to care with telehealth. Furthermore, no patient felt that telehealth would lead to a decrease in their health. The majority of patients $(90 \%)$ felt that telehealth would have either no effect or positive effect on their relationship with the CF care team, and $80 \%$ indicated support for future telehealth implementation at the $\mathrm{CF}$ center. There was also a moderate degree of interest toward telehealth education, with $35 \%$ of respondents indicating interest in telehealth family and patient education programs and $45 \%$ indicating interest in telehealth group education programs (Table 5).

\section{Discussion}

Although telehealth has been utilized previously in medicine in limited contexts, the onset of the coronavirus (COVID-19) pandemic has prompted health systems around the world to rapidly phase into telehealth on a scale never before seen. To help maintain social distancing while still providing medical care, restrictions on telehealth have been relaxed, and though this has helped increase telemedicine usage, many health systems including VCU were quickly overwhelmed by the sheer volume of patients and lack of guidance on how best to efficiently treat them remotely. To ameliorate these issues, VCU Adult CF Center participated in the Cystic Fibrosis Learning Center (CFLN) COVID-19 


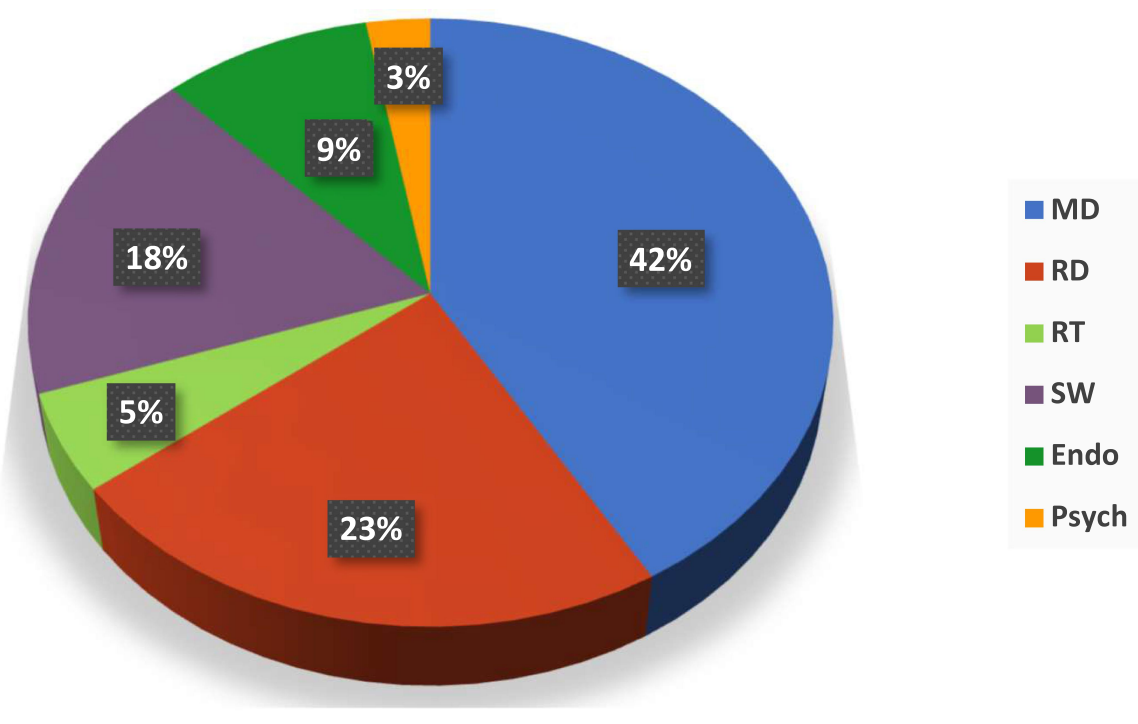

Figure 4 Percentage of virtual visits completed by different care team members (CTMs) at VCU Adult CF Center between April I and June $30,2020$.

Telehealth Innovation Lab (iLab) to design and refine a working model on using telehealth to treat patients with $\mathrm{CF}$.

Toward that end, we collected data from patients regarding satisfaction and limitations with the current telehealth model. Overall, patients indicated a positive reception with telehealth, specifically approving of the increased accessibility of care and the vast majority noting little to no negative effects on their health or patient-provider

Table 5 Results Collected from a Cross-Sectional Patient Survey Administered to Patients ( $N=20)$ Seen Through Virtual Visits at VCU Adult CF Center Between the Dates of April I and June 3, 2020

\begin{tabular}{|c|c|c|c|}
\hline Assessment & Yes & No & Undecided \\
\hline $\begin{array}{l}\text { Do you feel there will be improved } \\
\text { access to care with telehealth? }\end{array}$ & 20 & 0 & 0 \\
\hline $\begin{array}{l}\text { Do you feel that telehealth will lead to } \\
\text { a decrease in your health? }\end{array}$ & 0 & 19 & I \\
\hline $\begin{array}{l}\text { Do you feel telehealth will lead to } \\
\text { a decrease in time for clinic visits? }\end{array}$ & 10 & 8 & 2 \\
\hline $\begin{array}{l}\text { Do you think telehealth visits will have } \\
\text { a positive impact on your overall health? }\end{array}$ & 17 & I & 2 \\
\hline $\begin{array}{l}\text { Does geographic proximity to clinic play } \\
\text { a role your interest in telehealth? }\end{array}$ & 12 & 8 & 0 \\
\hline $\begin{array}{l}\text { Are you interested in a telehealth } \\
\text { patient and family education program? }\end{array}$ & 7 & 12 & I \\
\hline $\begin{array}{l}\text { Do you think we should implement } \\
\text { telehealth visits for the CF clinic? }\end{array}$ & 16 & 2 & 2 \\
\hline $\begin{array}{l}\text { Are you interested in group education } \\
\text { visits (eg airway clearance teaching or } \\
\text { nutrition education)? }\end{array}$ & 9 & 11 & 0 \\
\hline
\end{tabular}

relationship. This may be in part due to current events already limiting in-person contact overall, but the point still stands that the vast majority of survey participants responded positively to telehealth, indicating that this may continue to provide an accessible and preferable form of treatment even after the current health crisis.

However, we have noted several limitations to our current telehealth model. One challenge, which has been experienced by our center as well as in many areas of medicine as telehealth has been implemented, has been the procurement of pulmonary function tests (PFTs), cultures, labs, and imaging data. Results of PFTs, labs, culture, and imaging studies are essential tools that guide diagnosis and treatment and are especially important in the care of CF patients, in which population frequent monitoring is recommended by the CFF as standard of care. Provision of medical services remotely over telehealth has made it challenging to attain these studies, which normally would be done efficiently at the clinic on the same day as the visit. Strategies must be devised to allow efficient collection of these monitoring studies when patients are not visiting the clinic inperson in a manner that limits the risk of exposure to the virus.

In an attempt to overcome this challenge, our clinic has begun to implement at-home collection of sputum culture samples which are then mailed by the patient to the lab for processing. This intervention is newly implemented in our center and continues to be modified, so its success cannot yet be evaluated. Labs have been collected in the patients' 
homes through home-health nursing agencies, and imaging studies have been coordinated by the radiology department outside of our clinic. Similarly, there has been increased reliance on home spirometry for collection of PFT data, which are then communicated by the patient to the clinic. Electronic home spirometers have been shown to be equivalent to laboratory-based spirometry and more affordable, ${ }^{23,24}$ and the benefits of weekly home spirometry have been discussed as an alternative to quarterly inperson measurements for $\mathrm{CF}^{25}$ Future studies will need to assess the effectiveness of these methods and explore alternative solutions.

One other limitation was simply a lack of participation from patients. With only 20 out of 60 patients responding to the survey, any conclusions we can draw from the results are innately limited as the remainder of our patients may have differing views. As such, in future studies this issue may be mitigated by incentivizing patients to complete surveys either through raffles or gift cards or some other form of compensation.

Another limitation to the provision of CF care through telehealth may be access to appropriate technologies required for a successful virtual visit. Patients may lack access to high-speed internet connectivity or internetenabled devices that restrict their ability to take part in telehealth appointments. This issue is exacerbated by the fact that such limited access to the internet is often in rural regions that would most benefit from telehealth, where approximately a quarter of the population lacks broadband internet, ${ }^{26}$ which has the potential to widen the gap of healthcare disparities. One possible solution is to have clinics provide patients who lack internet access mobile data "hotspot" devices which can provide limited internet access. However, on a global or even national scale, such a plan may be excessively costly and shortsighted; for any lasting resolution, a joint effort would need to be made by policymakers, internet service providers, and medical institutions. Additionally, some patients may have limited proficiency with computer use or use of the Zoom video conferencing platform, which may hinder their ability to participate in a virtual appointment, although this could be resolved with proper training in most cases.

Likewise, the health systems themselves also may not have the technological capabilities to manage a high volume of telehealth appointments. The VCU Adult CF Center currently only has one virtual room in which to treat patients, which has resulted in a backup of appointments. These virtual meeting rooms must also be HIPAA- compliant to protect patient information, and any multidisciplinary communication in the care process must be done over HIPAA-compliant chatrooms. Building a robust IT expertise that is able to set up these capabilities and provide support for technology failures is essential. Expanding such technology capabilities will ensure effectiveness and sustainability of the telehealth model. Other areas of improvement include optimizing home and remote measurements such as pulse oximetry and blood pressure through electronic stethoscope technology, as well as recruiting home nursing services to provide home chest X-rays and other measures and, lastly, designing and providing hybrid care when possible to allow more intensive care as needed. These options are currently being explored by the VCU Adult CF Center and should be a point of future study.

As such, while the data may currently suggest positive reception toward telehealth and encourage continued usage even beyond this pandemic, more work is required to help improve the efficiency and accessibility of care options. Further studies may explore the provision of group or family-based education programs via telehealth, as our survey data anticipated patient interest in telehealth and this intervention has the potential to increase patient selfefficacy.

\section{Disclosure}

Caitlin Womack, Ruhan Farsin, and Mahsa Farsad have received no financial compensation for this review article. They have no conflicts of interest with any companies or organizations whose products or services may be discussed in this article. Nauman Chaudary reports grants from CF Foundation during the conduct of the study. The authors report no other conflicts of interest in this work.

\section{References}

1. Ronan NJ, Elborn JS, Plant BJ. Current and emerging comorbidities in cystic fibrosis. Presse Med. 2017;46(6 Pt 2):e125-e138. doi:10.1016/j. lpm.2017.05.011

2. Yankaskas JR, Marshall BC, Sufian B, Simon RH, Rodman D. Cystic fibrosis adult care: consensus conference report. Chest. 2004;125(1 Suppl):1S-39S. doi:10.1378/chest.125.1_suppl.1S

3. Johnson C, Butler SM, Konstan MW, Morgan W, Wohl ME. Factors influencing outcomes in cystic fibrosis: a center-based analysis. Chest. 2003;123(1):20-27. doi:10.1378/chest.123.1.20

4. Sullivan JS, Mascarenhas MR. Nutrition: prevention and management of nutritional failure in cystic fibrosis. J Cyst Fibros. 2017;16(Suppl 2):S87-S93. doi:10.1016/j.jcf.2017.07.010

5. Frost F, Dyce P, Ochota A, et al. Cystic fibrosis-related diabetes: optimizing care with a multidisciplinary approach. Diab Metab Syndr Obes. 2019;12:545-552. doi:10.2147/DMSO.S180597 
6. "About Cystic Fibrosis." Cystic fibrosis foundation. Available from: https://www.cff.org/What-is-CF/About-Cystic-Fibrosis/. Accessed July $8,2020$.

7. Smith AC, Thomas E, Snoswell CL, et al. Telehealth for global emergencies: implications for coronavirus disease 2019 (COVID-19). J Telemed Telecare. 2020;26(5):309-313. doi:10.1177/ 1357633X20916567

8. Fisk M, Livingstone A, Pit SW. Telehealth in the context of COVID-19: changing perspectives in Australia, the United Kingdom, and the United States. J Med Internet Res. 2020;22(6): e19264. doi:10.2196/19264

9. Contreras CM, Metzger GA, Beane JD, Dedhia PH, Ejaz A, Pawlik TM. Telemedicine: patient-provider clinical engagement during the covid-19 pandemic and beyond [published online ahead of print, 2020 May 8]. J Gastrointest Surg. 2020;1-6. doi:10.1007/ s11605-020-04623-5

10. Farfour E, Picard C, Beaumont L, et al. COVID-19 in lung-transplanted and cystic fibrosis patients: be careful. J Cyst Fibros. 2020;19(3):e16-e17. doi:10.1016/j.jcf.2020.03.021

11. Saiman L, Siegel J. Cystic fibrosis foundation consensus conference on infection control participants. Infection control recommendations for patients with cystic fibrosis: microbiology, important pathogens, and infection control practices to prevent patient-to-patient transmission. Am J Infect Control. 2003;3:S1-S62.

12. Neufeld JD, Doarn CR, Aly R. State policies influence medicare telemedicine utilization. Telemed J E Health. 2016;22(1):70-74. doi:10.1089/tmj.2015.0044

13. Mehrotra A, Jena AB, Busch AB, Souza J, Uscher-Pines L, Landon BE. Utilization of telemedicine among rural medicare beneficiaries. JAMA. 2016;315(18):2015-2016. doi:10.1001/jama.20 16.2186

14. Reed ME, Huang J, Graetz I, et al. Patient characteristics associated with choosing a telemedicine visit vs office visit with the same primary care clinicians. JAMA Netw Open. 2020;3(6):e205873. doi:10.1001/jamanetworkopen.2020.5873

15. Barnett ML, Ray KN, Souza J, Mehrotra A. Trends in telemedicine use in a large commercially insured population, 2005-2017. JAMA. 2018;320(20):2147-2149. doi:10.1001/jama.2018.12354

16. Rodger S, Kenton O. "Exploring the potential for technology to improve cystic fibrosis care provision.". Proc ACM HumanComputer Interaction. 2019;3:1-26. doi:10.1145/3359223
17. Bell SC, Mall MA, Gutierrez H, et al. The future of cystic fibrosis care: a global perspective [published correction appears in lancet respir med. 2019 Dec;7(12):e40]. Lancet Respir Med. 2020;8 (1):65-124. doi:10.1016/S2213-2600(19)30337-6

18. Gur M, Nir V, Teleshov A, et al. The use of telehealth (text messaging and video communications) in patients with cystic fibrosis: A pilot study. J Telemed Telecare. 2017;23(4):489-493. doi:10.117 7/1357633X16649532

19. Wood J, Mulrennan S, Hill K, Cecins N, Morey S, Jenkins S. Telehealth clinics increase access to care for adults with cystic fibrosis living in rural and remote Western Australia. J Telemed Telecare. 2017;23(7):673-679. doi:10.1177/1357633X16660646

20. Shigekawa E, Fix M, Corbett G, Roby DH, Coffman J. The current state of telehealth evidence: a rapid review. Health Aff. 2018;37 (12):1975-1982. doi:10.1377/hlthaff.2018.05132

21. Isasti G, Fernández JFD. Comparison of telehealth and traditional face-to-face model during COVID-19 pandemic [published online ahead of print, 2020 Sep 30]. Med Clin. 2020. doi:10.1016/j. medcle.2020.06.020

22. Compton M, Soper M, Reilly B, et al. A feasibility study of urgent implementation of cystic fibrosis multidisciplinary telemedicine clinic in the face of covid-19 pandemic: single-center experience [published online ahead of print, 2020 Apr 30]. Telemed $J$ E Health. 2020. doi:10.1089/tmj.2020.0091.

23. Tagliente I, Trieste L, Solvoll T, Murgia F, Bella S. Telemonitoring in cystic fibrosis: a 4-year assessment and simulation for the next 6 years. Interact J Med Res. 2016;5(2):e11. doi:10.2196/ijmr.5196.

24. Zhou P, Yang L, Huang YX. A smart phone based handheld wireless spirometer with functions and precision comparable to laboratory spirometers. Sensors. 2019;19(11):2487. doi:10.3390/s19112487

25. Shakkottai A, Kaciroti N, Kasmikha L, Nasr SZ. Impact of home spirometry on medication adherence among adolescents with cystic fibrosis. Pediatr Pulmonol. 2018;53(4):431-436. doi:10.1002/ppul. 23950

26. Eighth broadband progress report; August 23, 2012. Available from: https://www.fcc.gov/reports-research/reports/broadband-progressreports/eighth-broadband-progress-report. Accessed August 08, 2020.
International Journal of General Medicine

\section{Publish your work in this journal}

The International Journal of General Medicine is an international, peer-reviewed open-access journal that focuses on general and internal medicine, pathogenesis, epidemiology, diagnosis, monitoring and treatment protocols. The journal is characterized by the rapid reporting of reviews, original research and clinical studies across all disease areas. The manuscript management system is completely online and includes a very quick and fair peer-review system, which is all easy to use. Visit http://www.dovepress.com/ testimonials.php to read real quotes from published authors. 